\title{
Frontières
}

\section{Marie-Hélène Encrevé-Lambert, La mort, Paris, Bayar, 1999, 138 pages}

\section{Pierre-Alexandre Poirier}

Volume 13, numéro 1, automne 2000

URI : https://id.erudit.org/iderudit/1074259ar

DOI : https://doi.org/10.7202/1074259ar

Aller au sommaire du numéro

Éditeur(s)

Université du Québec à Montréal

ISSN

1180-3479 (imprimé)

1916-0976 (numérique)

Découvrir la revue

Citer ce compte rendu

Poirier, P.-A. (2000). Compte rendu de [Marie-Hélène Encrevé-Lambert, La mort, Paris, Bayar, 1999, 138 pages]. Frontières, 13(1), 83-84.

https://doi.org/10.7202/1074259ar

Ce document est protégé par la loi sur le droit d'auteur. L'utilisation des services d'Érudit (y compris la reproduction) est assujettie à sa politique d'utilisation que vous pouvez consulter en ligne.

https://apropos.erudit.org/fr/usagers/politique-dutilisation/
Cet article est diffusé et préservé par Érudit.

Érudit est un consortium interuniversitaire sans but lucratif composé de l’Université de Montréal, l'Université Laval et l'Université du Québec à Montréal. Il a pour mission la promotion et la valorisation de la recherche. https://www.erudit.org/fr/ 
nés, mais aussi des soignants, des enseignants, des travailleurs sociaux, pour ne nommer qu'eux. On y trouve résumés les points essentiels de la dynamique du deuil et explorées les significations de la disponibilité adulte à l'endroit des enfants qui vont perdre et perdent; le partage des émotions et sentiments, et surtout la question de la vérité, des réassurances de noncontagiosité, de non-abandon comme de non-responsabilité dans la mort de l'autre, et enfin de nonoubli de ce dernier, par divers gestes, dont l'écriture. Le travail des groupes de soutien est également décrit.

Enfin, la finale, en proposant de nouvelles ouvertures, trace finement le portrait si paradoxal des enfants en deuil et leur besoin de réparation, une fois devenus adultes, et nous offre dix-huit propositions ou aphorismes, pour quiconque voudrait par là aborder l'ouvrage...

Voilà donc un aperçu d'une œuvre réelle, sertie de considérations astucieuses tenant lieu de comparaisons nuancées entre le deuil chez l'enfant et chez l'adulte. Si on y trouve plusieurs reprises de l'ouvrage précédent de M. Hanus (1994), c'est avec encore plus d'éclat intellectuel et communicatif. Et l'apport de B. M. Sourkes (en deux chapitres) ne manque pas d'envergure clinique qui stimule le sens du questionnement. Bref, cette humanité à la fois touchante et intellectuellement fort vivace arrive au lecteur comme un vent porteur de bénéfices pour les enfants et tous les enfants, puisque par le biais de la pédagogie du deuil, nous risquons de mieux comprendre les jeunes affligés, mais aussi chacun et chacune de ceux que nous côtoyons.

\section{Luce Des Aulniers}

Un sens complémentaire en psychiatrie tiendrait dans la propension du Moi à croire que les histoires qu'il se raconte sont réelles; dans le langage courant, l'expression renvoie à la umanie des grandeurs); les trois acceptions ont trait à cet enflement du sentiment de puis sance, qui n'est pas sans rappeler le sentiment infantile de toute-puissance. perdurant à l'âge adulte et qui, d'autre part pourrait être avivé par la mentalite de croissance et de quête de prestige à tout prix.

\section{Daniel Oppenheim}

\section{Dialogues avec les enfants sur la vie et la mort \\ Paris, Seuil, 2000, 204 pages.}

Daniel opdenheim

DIALOGUES

AVEC LES

ENFANIS

SUR LA VIE

ET LA MORT

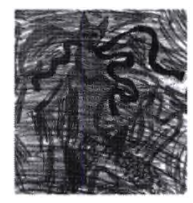

Seoll

"Fictions exemplaires. "

"Plutôt que de donner des conseils toujours schématiques, j'ai préféré raconter des histoires au long desquelles apparaissent des repères et des points d'appui que le lecteur pourra utiliser pour engager librement, à sa manière, le dialogue avec l'enfant et en suivre le déroulement. " "Dialoguer n'est pas donner une leçon mais s'engager personnellement. "Les trois citations mises en exergues de cette recension sont extraites du premier chapitre du volume de Daniel Oppenheim et condensent les propos que je veux développer ici. À partir de "vingt-cinq années de pratique psychothérapeutique et psychanalytique, à Saint-Denis, Saint-Lô, Paris et au sein du département d'oncologie pédiatrique de l'institut Gustave Roussy à Villejuif ", l'auteur a construit, condensé et amalgamé une série de douze fictions exemplaires. Inscrivant son expérience clinique au plus près des processus conscients, préconscients ou inconscients qui se retrouvent dans la construction d'un dialogue entre les parents et les enfants autour du thème de la mort, Daniel Oppenheim permet de faire formuler aux enfants de ses fictions exemplaires les questions progressives qu'ils éprouvent face à la mort. Le cheminement y est juste et les embûches, sur le chemin des réponses recherchées, signifiées. La présence de phrases incises, comme un commentaire en aparté, relance le propos, donne au dialogue l'inflexion d'une couleur thérapeutique qui m'est apparue idéalisante. C'est alors qu'il faut se rappeler que l'auteur a " préféré raconter des histoires au long desquelles apparaissent des repères et des points d'appui que le lecteur librement, pourra utiliser pour engager à sa manière, le dialogue avec l'enfant et en suivre le déroulement. "

Je crois cependant que la pertinence et l'appoint des propos que l'auteur prête aux parents pour rejoindre le questionnement de leurs enfants a quelque chose de " très " exemplaire. C'est pour moi comme si, au cours de ces dialogues, les parents avaient tous réussi des introspections pacifiantes intériorisées sur le grand thème de la mort. Même lorsqu'une difficulté à toucher le thème est évoquée, par exemple de l'ordre d'une contre-réaction plus personnelle, l'auteur fait trouver aux parents le moyen de dénouer l'impasse. Cette formule a le mérite de montrer les solutions de nature thérapeutique. Elle n'accompagne le parent dans son propre questionnement sur la mort.

L'auteur nous a bien dit que dialoguer n'est pas donner une leçon mais s'engager personnellement, j'ai l'impression qu'une partie de l'engagement personnel a été escamotée ou mise de côté.

Ce livre ne touche pas la mort d'un parent de l'enfant. L'auteur nous indique que selon lui ce thème nécessiterait un volume à lui seul et il a choisi de ne pas le traiter.

Dans un style vif, de la confrontation du "Deuil d'un animal familier " (chapitre 2) à "La fin de la vie du grand-père " (chapitre 11) l'auteur utilisera des situations proto typiques que la clinique lui a fait connaître, auxquelles il adjoindra des situations sociales, par exemple "L'enfant face aux images de la mort au cinéma et à la télévision " (chapitre 3) ou " La médecine et la mort dans les familles " (chapitre 9) pour exposer de quelle façon l'enfant s'approche de la mort et l'apprivoise. Chacun des chapitres a son identité propre et peut être consulté individuellement. C'est ainsi qu'on apprend dans le chapitre sur " La médecine et la mort dans les familles " qu'une loi française autorise le prélèvement d'organes à moins que la personne s'inscrive " sur un registre national de refus". L'auteur ouvre ainsi la voie à un questionnement éthique.

"La fin de vie du grand-père " reçoit une attention particulière. Cette situation est plus fréquente dans la réalité des enfants et la fiction exemplaire mise ici en mots nous fait connaître les interroga- tions que suscite le processus de cette mort pour une petite fille et les réponses proposées à l'occasion d'un dialogue avec ses parents.

En conclusion, je dirai que le partage par le lecteur de l'expérience condensée d'un clinicien sur le thème de la vie et la mort trouve ici des points d'ancrage suffisants pour que le lecteur " engage à sa manière le dialogue avec l'enfant et en suive le déroulement ", malgré le rôle idéalisant que l'auteur fait jouer aux parents.

\section{Yvon Forget}

Marie-Hèlène Encrevé-Lambert

\section{La mort}

Paris, Bayar, 1999, 138 pages.

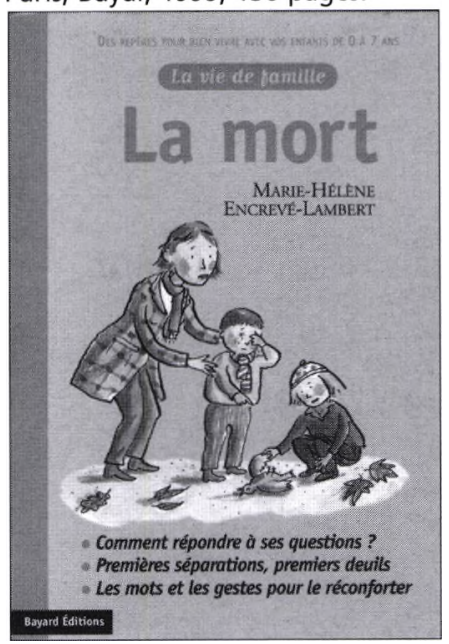

Marie-Hélène Encrevé-Lambert présente, dans cette jeune collection publiée chez Bayard, une somme concise d'informations essentiellement psychanalytiques et anthropologiques sur l'expérience de la mort chez l'enfant. L'auteur retient la thèse selon laquelle nombre de sociétés occidentales contemporaines éprouvent un important malaise devant la mort, compliquant sérieusement la capacité des parents à accompagner les enfants dans la surprise et la souffrance d'une perte. À une époque où $75 \%$ des décès ont lieu derrière les murs d'un hôpital, les enfants et les adultes ne côtoient plus la mort comme autrefois. Ils ne sont plus familiarisés avec elle. Aussi, le véritable problème n'est pas de parler de la mort aux enfants - nous ne savons rien d'elle - mais plutôt de savoir recueillir leurs questionnements, accueillir leurs découvertes et accompagner leurs angoisses. En un mot, les adultes doivent savoir se comporter pour que les enfants puissent continuer à partager leurs interrogations (p. 18). Dans cet ouvrage, l'auteur, qui s'adresse aux parents, 
n'offre pas de "recettes" pour discuter de ce sujet délicat, mais invite plutôt à une réflexion sur le thème de la mort à partir de son expérience de psychanalyste auprès des enfants et des adultes (p. 15). Nous avons choisi de découper cette réflexion en trois temps : le rapport spécifique de l'homme avec la mortalité, l'expérience de la souffrance et de la mort chez l'enfant et la discussion entre parents et enfants sur la mort.

Pour Encrevé-Lambert, la conscience de la mort, qui est le propre de l'homme, est inséparable du langage (p. 30). Le langage permet de nommer les émotions et les sentiments confus que l'enfant connaît dès sa naissance, c'est-àdire dès la séparation d'avec la mère. Il s'agit de sentiments de manques comme la faim, le froid et la douleur liés à une fragilité et à une mortalité qu'il découvre graduellement. En nommant ces émotions et en apportant la réponse matérielle appropriée, la mère va contribuer à leur donner un sens, et ainsi permettre à l'enfant de s'inscrire dans le langage. Un langage qui donne sens à son existence. Le langage permet de penser la mortalité et l'absence, et par conséquent de rendre présente cette absence. Mais, dit-elle, puisque le langage est toujours inadéquat, il ne peut rendre compte d'une émotion liée à un manque lorsque cette émotion est trop intense. Ce vide que creuse le langage est souvent exprimé par des expressions telles que : " II n'y a pas de mots pour dire ma peine"

Contrairement à la croyance de bien des parents, le jeune enfant a déjà une expérience du deuil et certaines connaissances sur la mort. Son expérience du deuil se fait avec la séparation de la mère. Cette expérience première sert de modèle aux séparations futures (p. 31). Pour Encrevé-Lambert, un jeune bébé de trois mois vit tout autant la souffrance qu'un adulte et s'organise psychiquement pour pouvoir la dépasser (p. 32). De même, bien avant d'être placé devant la mort d'un être cher, un jeune enfant a bien souvent fait la découverte de petits animaux morts dans la nature, ce qui lui procure un certain savoir sur la mortalité. Ces découvertes sont bien sûr des occasions de parler de la mort avec l'enfant qui a déjà des théories pour I'expliquer. Pour l'auteur, il s'agit moins de donner des réponses que de comprendre où en est l'enfant dans le développement de sa compréhension.

Ces discussions sont importantes. Elles aident, le jour venu, à aborder la difficile question d'une perte douloureuse. Dans de telles circonstances, l'enfant a besoin d'être lié aux adultes par la parole en partageant ses sentiments, mais aussi en assistant au rituel funéraire (p. 99) et en se faisant une idée du devenir du défunt (p.103). Les sentiments auxquels renvoie la mort sont multiples : insécurité, détresse, abandon, etc. II va de soi que la perte vécue est très différente selon qu'il s'agit d'un grandparent, du père, de la mère, du frère ou de la sœur (p. 56). Dans tous les cas, cette perte doit être mise en sens par la parole d'un adulte en qui l'enfant a confiance. L'enfant fait partie de la famille, et quel que soit son âge, la mort d'un proche le concerne. À ce titre, l'auteur souligne que si la mort d'un être proche est parfois vécue à travers un sentiment d'abandon, le silence malaisé des adultes redouble ce sentiment (p. 53). Alors que les parents cherchent souvent à protéger l'enfant d'une réalité qu'ils ne peuvent accepter, l'enfant veut être tranquillisé sur le fait d'être mortel et sur le danger de perdre l'amour du défunt. II s'agit là d'une rencontre qui n'arrive pas à se faire, et qui augmente l'angoisse devant la mort. Aussi, bouleversé par l'impossibilité de parler de la mort, certains enfants peuvent " se retirer de la communication " avec leurs parents (p. 49).

On ne peut que féliciter Encrevé-Lambert pour cet ouvrage clair et accessible au grand public. L'auteure, en outre, aide les adultes à réfléchir sur l'accompagnement d'un enfant dans son cheminement vers l'appropriation de la mortalité. La mort, écrit l'auteur en conclusion, " ne s'apprend pas et personne n'a plus de compétence qu'un autre pour en parler. Si chaque adulte a le courage (il en faut) de se poser la question de sa mort et de la mort de ceux qu'il aime ou a aimés, il saura faire face aux interrogations de son enfant " (p. 129).

Pierre-Alexandre Poirier

Marie-Frédérique Bacqué et Michel Hanus

\section{Le deuil}

Coll. "Que sais-je ? " no 3558, Paris, PUF, 2000, 127 pages.

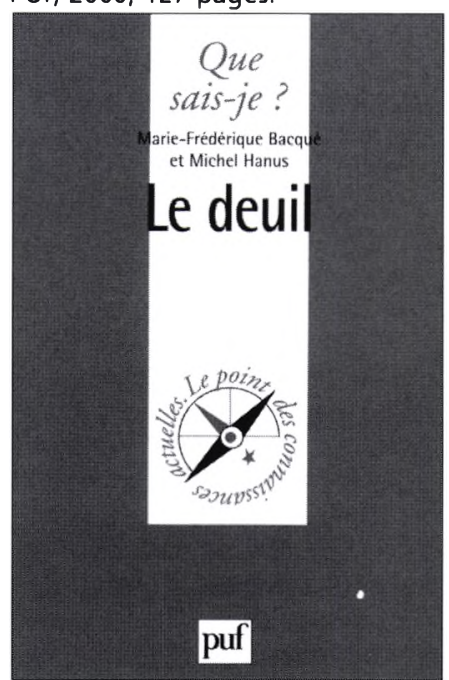

En 1915, dans son livre intitulé Deuil et mélancolie, Freud écrivait que "le deuil est la réaction habituelle à la perte d'une personne aimée ou d'une abstraction mise à sa place, la patrie, un idéal, la liberté, etc. ". Selon cette définition, il $y$ a donc des deuils qui ne sont pas liés à la mort mais à la perte, comme le deuil de la langue pour celui qui émigre dans un pays étranger, le deuil d'un emploi perdu, le deuil d'un divorce non désiré, etc. Le mot " deuil " est susceptible d'une extension encore plus grande, car s'il y a deuil chaque fois qu'il y a perte, il y a donc deuil toujours. En effet, nul n'a jamais pu voir tous ses désirs être complètement satisfaits. " Nous ne savons renoncer à rien ", écrivait encore Freud dans ses Essais de psychanalyse appliquée et c'est pourquoi le deuil est ce contre quoi le plaisir et le désir échouent. Dit autrement, le temps est le deuil même de l'être. Par conséquent, la mort n'est pas que le dernier instant de la vie; elle est ce qui, dès le début, empêche de naître et de grandir; elle est ce processus de destruction qui est inhérent à toute vie.

Toutefois, dans le présent livre, le deuil a un sens beaucoup plus restrictif : il ne vise que l'ensemble des réactions que la mort, ce terme de la vie, entraîne. En outre, l'ouvrage n'aborde la question du deuil qu'à l'aide des nouvelles approches psychologique et sociale de la perte.

A cette double limite, il faut ajouter qu'il peut se réduire à l'étude de trois grands thèmes: les représentations de la mort et du deuil en Occident (p. 3-19), les adultes et le deuil (p. 20-77), les enfants et le deuil (p. 78-122).

Dans l'avant-propos (p. 3-10) et le premier chapitre (p. 11-19), les auteurs nous rappellent l'importance des cérémonies rituelles et nous expliquent les principales causes de la désaffection des pratiques sociales de deuil : transgression des rites de mort à grande échelle (massacre des guerres mondiales et génocides), urbanisation accélérée, évolution démographique, institutionnalisation et professionnalisation de la mort, désenchantement du monde, etc.

Le deuxième grand thème, qui a pour objet l'adulte et le deuil, se subdivise lui-même en sept parties. D'une part, les auteurs exposent quelques définitions du deuil ( $p$. 20-26). D'autre part, ils présentent le déroulement du deuil normal : état de choc ou blocage somatopsychique, recherche et régression, agressivité et colère, chagrin ou dépression dynamique sur le plan somatique, intellectuel et affectif, et acceptation (p. 27-35). Puis, suit une analyse psychologique du tra vail de deuil qui oscille constamment entre refus, régression, culpabilité, acceptation et réactivation (p. 36-42). Dans le cas d'un deuil " normal ", ces étapes respectent habituellement un ordre déterminé et aboutissent toujours au même résultat final : l'intériorisation du défunt par le sujet. Bien entendu, diverses complications au cours du travail de deuil peuvent survenir (p. 43-50). Le deuil peut être inhibé ou différé. Au contraire, il peut devenir chronique. Parmi les facteurs expliquant ces complications du deuil, il y a la relation préexistante à la perte, les circonstances atypiques du décès, la manière dont le décès est annoncé, l'âge, la santé et la situation professionnelle de l'endeuillé, les deuils répétés, l'absence de rites communautaires et la mauvaise qualité d'écoute de l'entourage. Les complications sont parfois si graves qu'elles peuvent même provoquer certaines pathologies du deuil (p. 51-59). Parmi celles-ci, les auteurs analysent rapidement les deuils hystérique, obsessionnel, maniaque, mélancolique, traumatique, etc. Les diffèrents deuils particuliers sont également examinés : le deuil de l'enfant, le deuil dans des conditions traumatiques (catastrophes, survivants des camps de la mort, etc.), le deuil et les maladies chroniques (sida, cancer, etc.) et le deuil des soignants (p. 60-71). Finalement, une brève réflexion sur l'accompagnement des personnes 